\title{
Retinal microstructural abnormalities in central serous chorioretinopathy and polypoidal choroidal vasculopathy.
}

\section{$\operatorname{AUTHOR}(\mathrm{S})$ :}

Ooto, Sotaro; Tsujikawa, Akitaka; Mori, Satoshi; Tamura, Hiroshi; Yamashiro, Kenji; Otani, Atsushi; Yoshimura, Nagahisa

\section{CITATION:}

Ooto, Sotaro ...[et al]. Retinal microstructural abnormalities in central serous

chorioretinopathy and polypoidal choroidal vasculopathy.. Retina 2011, 31(3): 527-534

\section{ISSUE DATE:}

2011-03

\section{URL:}

http://hdl.handle.net/2433/156787

\section{RIGHT:}

(c) The Ophthalmic Communications Society, Inc.; This is not the published version. Please cite only the published version.; この論文は 出版社版でありません。引用の際には出版社版をご確認ご利用くださ い。 


\section{RETINAL MICROSTRUCTURAL ABNORMALITIES IN CENTRAL SEROUS CHORIORETINOPATHY AND POLYPOIDAL CHOROIDAL VASCULOPATHY}

Short title: Retinal microstructure in CSC and PCV

SOTARO OOTO, MD, AKITAKA TSUJIKAWA, MD, SATOSHI MORI, MD, HIROSHI TAMURA, MD, KENJI YAMASHIRO, MD, ATSUSHI OTANI, MD, NAGAHISA YOSHIMURA, MD

Author Affiliations: Department of Ophthalmology and Visual Sciences, Kyoto University Graduate School of Medicine, Kyoto, Japan

Support: This research was supported in part by a Grant-in-Aid for Scientific Research (21791679) from the Japan Society for the Promotion of Science (JSPS).

Conflict of Interest: None of the authors have any financial/conflict of interest to disclose.

Corresponding author: Sotaro Ooto, MD

Department of Ophthalmology and Visual Sciences, Kyoto University Graduate School of Medicine, 54 Kawahara-cho, Shogoin, Sakyo-ku, Kyoto 606-8507, Japan

Telephone: +81-75-751-3248; Facsimile: +81-75-752-0933 
Ooto et al. Page 2

E-mail: ohoto@kuhp.kyoto-u.ac.jp

Key Words: central serous chorioretinopathy, optical coherence tomography, polypoidal choroidal vasculopathy

\section{Summary Statement}

By using speckle-noise-reduced spectral-domain optical coherence tomography, we compared retinal structures in polypoidal choroidal vasculopathy (PCV) and central serous chorioretinopathy (CSC) and observed more severe retinal alterations in PCV than in CSC due to intraretinal infiltration of fibrin and hemorrhage.

This manuscript contains 6 online-only supplemental figures. (Supplemental Figures 1-6) 
Ooto et al. Page 3

Abstract

Purpose: To compare retinal morphologic alterations in eyes with polypoidal choroidal vasculopathy (PCV) and central serous chorioretinopathy (CSC) using speckle-noise-reduced spectral domain optical coherence tomography (SD-OCT).

Methods: We retrospectively reviewed 63 eyes of 62 patients with active PCV and 38 eyes of 38 patients with active CSC. Patients underwent fundus photography, angiography, and speckle-noise-reduced SD-OCT examinations, and retinal morphologic alterations were evaluated.

Results: Cystoid macular edema, lipid deposits, subretinal hemorrhage, and hemorrhagic pigment epithelial detachment (PED) were not seen in any eye with CSC, but were seen in eyes with PCV. In PCV, mean visual acuity was significantly poorer in eyes with fibrin infiltration $(P=0.027)$ or hemorrhagic infiltration $(P=0.002)$ in the fovea than in eyes without fibrin or hemorrhagic infiltration.

Conclusion: Differentiating factors between PCV and CSC noted on SD-OCT include a lack of cystoid macular edema, lipid deposition, subretinal hemorrhage, and hemorrhagic PED in eyes with CSC, which makes SD-OCT helpful in differentiating CSC from PCV. More severe retinal alterations were seen in PCV than in CSC due to infiltration of fibrin and hemorrhage in the outer retina, which also correlated with poorer vision. 


\section{Introduction}

Polypoidal choroidal vasculopathy (PCV) is characterized by multiple, terminal reddish-orange nodules and a complex network of vessels, and it often causes serous retinal detachment $(\mathrm{SRD})$ or pigment epithelial detachment (PED). ${ }^{1-6}$ While its pathogenesis is not yet fully understood, PCV originates from an abnormality of the inner choroidal vessels and is presumed to be a variant of choroidal neovascularization (CNV).

Central serous chorioretinopathy (CSC) is characterized by SRD in the macula, often in association with small serous PEDs and retinal pigment epithelial atrophy. ${ }^{7,8}$ Evaluations using indocyanine green angiography (IA) show multifocal islands of inner choroidal staining, suggesting that exudative changes within the inner choroid are the primary event in CSC. ${ }^{9-14}$

Some cases of PCV have clinical, fluorescein angiography (FA), IA, or tomographic findings that are similar to $\mathrm{CSC}^{3-5}$ Choroidal vascular hyperpermeability, reportedly a characteristic finding in CSC, might be involved in the pathogenesis of PCV. ${ }^{4}$ Both PCV and CSC are associated with SRD. However, most patients with CSC have good visual acuity despite macular detachment, whereas many patients with PCV experience decreased visual acuity. The detailed features of the retinal morphologic changes that cause different visual outcomes have, however, not been documented.

A new spectral-domain optical coherence tomography (SD-OCT) system, the Spectralis ${ }^{\mathrm{TM}} \mathrm{HRA}+\mathrm{OCT}$ (Heidelberg Engineering, Dossenheim, Germany), combines a confocal scanning laser ophthalmoscope (SLO) and SD-OCT and allows information obtained from FA, IA, and SD-OCT to be combined with the 
certainty of knowing the exact site of origin. In addition, by using an eye tracking system, this new SD-OCT system has solved the problem of motion artifacts that limit the confident detection of small changes. Combining eye tracking with multiple B-scan averaging technology ${ }^{15}$ permits the production of detailed, speckle-noise-reduced images of all the retinal layers. These technological advances permit more detailed observation of various macular diseases and should help to clarify the different pathological features of CSC and PCV. In the present study, we examined and compared the retinal structure in PCV and CSC by using the speckle-noise-reduced SD-OCT system.

\section{Methods}

For this observational case study, we retrospectively reviewed 38 consecutive eyes of 38 patients ( 31 men and 7 women) diagnosed with active CSC and 63 consecutive eyes of 62 patients (48 men and 14 women) diagnosed with active PCV who visited the Macular Service at Kyoto University Hospital, Kyoto, Japan, for the first time between November 2007 and October 2008. All patients were Japanese. All investigations adhered to the tenets of the Declaration of Helsinki, and the study was approved by the Institutional Review Board and the Ethics Committee at Kyoto University Graduate School of Medicine.

Only eyes with active CSC or active PCV were included. Active CSC consisted of acute, chronic, or recurrent CSC. Quiescent CSC or PCV, which was defined as an atrophic macula without leakage on FA, was excluded from this study. Eyes with a history of photocoagulation, photodynamic therapy, or vitreous surgery were also excluded. 
All patients underwent a comprehensive ophthalmologic examination, including measurement of best-corrected visual acuity (VA) and intraocular pressure, indirect ophthalmoscopy, slit-lamp biomicroscopy with a contact lens, fundus photography, and simultaneous FA and IA using a confocal laser scanning system (Spectralis ${ }^{\mathrm{TM}} \mathrm{HRA}+\mathrm{OCT}$ ). Retinal imaging was performed using the Spectralis ${ }^{\mathrm{TM}} \mathrm{HRA}+\mathrm{OCT}$. First, horizontal and vertical line scans through the fovea centralis were obtained at a $30^{\circ}$ angle-width, followed by radial scans centered at the fovea; finally, serial horizontal scans were obtained at a $30^{\circ}$ angle-width. At each location of interest on the retina, 12-50 SD-OCT images were acquired and averaged to reduce the speckle noise.

Features of the retinal structure on SD-OCT images were evaluated by 2 observers (SM, HT); they were both experienced in evaluating OCT images, were blinded to diagnosis and visual acuity, and worked independently. In the case of disagreement, the opinion of a third observer (SO) was invited, and the results were discussed until a consensus was reached. An unpaired $t$ test was used to compare patient characteristics and speckle-noise-reduced OCT findings. All statistical evaluations were performed using a commercially available software program (SPSS17; SPSS Inc., Chicago, IL). A P value of less than 0.05 was considered to be statistically significant.

\section{Results}

In the current study, we examined 38 eyes of 38 patients with CSC and 63 eyes of 62 patients with PCV. PCV patients were significantly older; the mean age $( \pm$ standard deviation) was $47.1 \pm 9.9$ years (range, 30 to 72 years) in patients with 
Ooto et al. Page 7

CSC and $73.9 \pm 7.9$ years (range, 59 to 92 years) in patients with PCV $(P<$ 0.001). The duration of symptoms ranged from 1 month to 4 years (median, 2.0 months) for CSC and from 1 month to 6 years (median, 3.0 months) for PCV. VA was significantly better in eyes with CSC than in eyes with PCV $(P<0.001)$. The mean best-corrected VA was 0.75 (0.13 logMAR) in eyes with CSC, ranging from 0.06 to 1.5 , and $0.34(0.47 \log M A R)$ in eyes with $P C V$, ranging from 0.02 to 1.5 .

By using speckle-noise-reduced SD-OCT, eyes with CSC or PCV were examined in detail for morphologic changes (Figures 1-3, Supplemental Figures 1-6). Patient characteristics and speckle-noise-reduced SD-OCT findings are shown in Table 1. SRD was observed in all the eyes with CSC and in 61 (97\%) eyes with PCV $(P=0.159)$. Although cystoid macular edema $(\mathrm{CME})$ was not seen in any eye with CSC, 14 eyes with PCV (22\%) showed CME $(P<0.001$, Supplemental Figure 1). CME was observed around the polypoidal lesions in 13 (93\%) of these 14 eyes, and above the branching vascular network, apparent with IA, in one eye (7\%). In addition, while no eye with CSC showed lipid deposition, it was observed in 49 eyes with PCV (78\%, $P<0.001)$. Upon SD-OCT examination, the lipid deposition appeared as hyperreflective dots distributed at any depth from the inner retina to the outer retina (Supplemental Figure 2).

Fibrin was frequently observed in eyes with either CSC or PCV. A hyperreflective shadow suggesting fibrin in the subretinal space was observed in 11 eyes with CSC (29\%) and in 25 eyes with PCV $(40 \%, P=0.272)$. In eyes with CSC, this hyperreflective shadow was detected around small PEDs, which corresponded to the leakage point seen on FA (Supplemental Figure 3). In some 
Ooto et al. Page 8

of these areas shed photoreceptor outer segment $(O S){ }^{16}$ was seen

(Supplemental Figure 3). In eyes with PCV, a hyperreflective shadow suggestive of fibrin was observed around the reddish-orange nodules. Swelling of the outer nuclear layer $(\mathrm{ONL})$ or dipping of the posterior layer of the retina was observed often within these lesions (Supplemental Figure 4). In 12 of the 25 eyes with PCV in which we observed fibrin (48\%), SD-OCT images revealed fibrin infiltration into the outer retina, as indicated by intense reflectivity, especially between the OS and the external limiting membrane (ELM) (Figure 1). This infiltration of fibrin into the fovea seemed to be associated with poor visual function (Table 2). Fibrin was observed beneath the fovea in 21 eyes with PCV. In eyes with fibrin infiltration in the fovea (Figure 1), the mean VA was $0.20(0.70$ logMAR, $n=8)$. In contrast, in eyes without intense reflectivity in the outer retina (Figure 2), mean VA was $0.44(0.36 \log M A R, \mathrm{n}=13)(P=0.027$, unpaired $t$ test). Four of 11 eyes with CSC and fibrin (36\%) showed fibrin infiltration in the outer retina, all of which was extrafoveal.

Subretinal hemorrhage was observed in 33 eyes with PCV (52\%) and no eyes with CSC $(P<0.001)$. In 24 of these 33 affected eyes $(73 \%)$, SD-OCT revealed that the hemorrhage had infiltrated the outer retina, as indicated by intense reflectivity, especially between the OS and ELM (Figure 3). This infiltration of hemorrhage into the fovea was significantly associated with poor VA (Table 3). In 30 eyes with PCV, subretinal hemorrhage was observed beneath the fovea. In eyes with infiltration of hemorrhage in the fovea (Figure 3), mean best-corrected VA was $0.19(0.73 \log M A R, n=21)$; in contrast, in eyes without intense reflectivity in the outer retina (Supplemental Figure 5), mean 
best-corrected VA was $0.47(0.33 \log M A R, n=9)(P<0.001$, unpaired $t$-test).

A hemorrhagic PED was observed in 17 eyes with PCV (27\%) and none of the eyes with CSC. In 5 of these 17 affected eyes (29\%), SD-OCT showed intense reflectivity due to infiltration of hemorrhage or exudate in the outer retina, especially from the OS to the ELM, just above the hemorrhagic PED (Supplemental Figure 6). In one eye with poor visual function (VA $=0.15)$, this intense reflectivity was seen in the fovea.

\section{Discussion}

The development of OCT has provided a better understanding of the mechanisms involved in CSC and PCV. ${ }^{17-27}$ Time-domain OCT has been used to identify increased thickening and granularity of the outer photoreceptor layer in the area of SRD in CSC. ${ }^{17,18}$ Matsumoto et $\mathrm{al}^{19}$ also used SD-OCT to demonstrate the elongation of photoreceptor outer segments and the decreased thickness of the outer nuclear layer in CSC. OCT imaging of eyes with PCV revealed sharp protrusions of the RPE with moderate inner reflectivity; the protrusions were associated with reddish-orange nodules seen on fundus photography. ${ }^{21,22}$ and a double-layered line at the level of RPE is associated with a branching vascular network in $\mathrm{PCV} .{ }^{23}$ However, to date, limited information is available on the retinas of eyes with PCV, and the differences between the retinal morphologic changes seen in CSC and PCV have not been documented.

Because good VA in the acute form of CSC indicates the apparent preservation of good receptor function, it can be assumed that the metabolic 
exchange between the RPE and photoreceptors is relatively well maintained. In contrast, many eyes with PCV experience decreased visual acuity upon separation of foveal photoreceptors from the RPE. In the present study, lipids and subretinal hemorrhage were seen only in eyes with PCV. Lipids were distributed from the inner retina to the outer retina, suggesting that fluids and blood plasma might move from the subretinal space to the neuroretina. In eyes with PCV, plasma constituents and exudative products are contained in the subretinal fluid, which may prevent metabolic exchange between the RPE and photoreceptors, thereby making it difficult to preserve receptor function. Another theory would be that PCV is associated with increased VEGF expression ${ }^{28}$ which also affects the retina by increasing vascular permeability allowing the lipid/plasma to be released from retina vessels. In addition, CME was seen only in eyes with PCV, suggesting that retinal alteration is more severe in eyes with PCV than in those with CSC.

The infiltration of fibrin or hemorrhage was seen in the outer retina, especially between the OS and the ELM, most of which was below the ELM. The ELM thus appears to act as a barrier to the spread of fibrin or hemorrhage. The zonula adherens between the Müller cells and photoreceptors at the base of the OS, which make up the ELM, has a very narrow angle. ${ }^{29}$ It is not sealed, like the zonula occludens of the RPE and retinal capillaries, but limits the movement of large molecules. Such large molecules do not diffuse freely across the retina but, rather, are blocked partially by the ELM. Accordingly, it is reasonable to assume that photoreceptor inner segments (IS) and OS may be damaged by fibrin products or hemorrhage in PCV. 
Infiltration of hemorrhage or of exudative products in the outer retina may influence visual function in PCV. In eyes with fibrin or hemorrhagic infiltration into the fovea, visual acuity was worse than in eyes without it. In eyes without infiltration of fibrin or hemorrhage into the outer retina, VA was relatively sustained, even if fibrin or subretinal hemorrhage were observed beneath the fovea. Photoreceptors, especially IS and OS, may be directly damaged by intraretinal infiltration of hemorrhage or inflammatory products. The high incidence of hemorrhage or exudative product infiltration may lead to a poor visual outcome in PCV compared with CSC.

Hemorrhagic PED may also influence visual function in PCV. In some eyes with PCV in which hemorrhagic PED was observed, intense reflectivity was noted in the outer retina, especially from the OS to the ELM, above the hemorrhagic PED. We suspect that the intense reflectivity observed in the outer retina is due to infiltration of hemorrhage or exudates from the PED into the outer retina. Breakdown of the barrier function of the RPE allows an influx of hemorrhage or exudates into the retina.

In conclusion, speckle-noise-reduced SD-OCT allows more detailed observation of retinal structures and helps clarify the pathologic features of PCV. Differentiating factors noted on SD-OCT between PCV and CSC include lack of cystoid macular edema, lipid deposition, subretinal hemorrhage, and hemorrhagic PED in eyes with CSC, which makes SD-OCT helpful in differentiating CSC from PCV. More severe retinal alterations were seen in PCV than in CSC due to infiltration of fibrin and hemorrhage in the outer retina, which correlated with poorer vision. 


\section{References}

1. Yannuzzi LA, Sorenson J, Spaide RF, Lipson B. Idiopathic polypoidal choroidal vasculopathy (IPCV). Retina 1990; 10:1-8.

2. Yannuzzi LA, Wong DW, Sforzolini BS, et al. Polypoidal choroidal vasculopathy and neovascularized age-related macular degeneration. Arch Ophthalmol 1999; 117:1503-1510.

3. Yannuzzi LA, Ciardella A, Spaide RF, et al. The expanding clinical spectrum of idiopathic polypoidal choroidal vasculopathy. Arch Ophthalmol $1997 ; 115: 478-485$.

4. Sasahara M, Tsujikawa A, Musashi K, et al. Polypoidal choroidal vasculopathy with choroidal vascular hyperpermeability. Am J Ophthalmol $2006 ; 142: 601-607$.

5. Yannuzzi LA, Freund KB, Goldbaum M, et al. Polypoidal choroidal vasculopathy masquerading as central serous chorioretinopathy. Ophthalmology 2000; 107:767-777.

6. Moorthy RS, Lyon AT, Rabb MF, Spaide RF, Yannuzzi LA, Jampol LM. Idiopathic polypoidal choroidal vasculopathy of the macula. Ophthalmology $1998 ; 105: 1380-1385$.

7. Yannuzzi LA, Shakin JL, Fisher YL, Altomonte MA. Peripheral retinal detachments and retinal pigment epithelial atrophic tracts secondary to central serous pigment epitheliopathy. Ophthalmology 1984; 91:1554-1572.

8. Levine R, Brucker AJ, Robinson F. Long-term follow-up of idiopathic central serous chorioretinopathy by fluorescein angiography. Ophthalmology 1989; 
96:854-859.

9. Guyer DR, Yannuzzi LA, Slakter JS, Sorenson JA, Ho A, Orlock D. Digital indocyanine green videoangiography of central serous chorioretinopathy. Arch Ophthalmol 1994; 112:1057-1062.

10. Piccolino FC, Borgia L. Central serous chorioretinopathy and indocyanine green angiography. Retina 1994; 14:231-242.

11. Scheider A, Nasemann JE, Lund OE. Fluorescein and indocyanine green angiographies of central serous choroidopathy by scanning laser ophthalmoscopy. Am J Ophthalmol 1993; 115:50-56.

12. Prunte C, Flammer J. Choroidal capillary and venous congestion in central serous chorioretinopathy. Am J Ophthalmol 1996; 121:26-34.

13. lida T, Kishi S, Hagimura N, Shimizu K. Persistent and bilateral choroidal vascular abnormalities in central serous chorioretinopathy. Retina 1999; 19:508-512.

14. Fujimoto H, Gomi F, Wakabayashi T, Sawa M, Tsujikawa M, Tano Y. Morphologic changes in acute central serous chorioretinopathy evaluated by Fourier-domain optical coherence tomography. Ophthalmology 2008; 115:1494-1500.

15. Sakamoto A, Hangai M, Yoshimura N. Spectral-domain optical coherence tomography with multiple B-scan averaging for speckle-noise-reduced imaging of retinal diseases. Ophthalmology 2008; 115:1071-1078.

16. Spaide RF. Autofluorescence from the outer retina and subretinal space: hypothesis and review. Retina 2008; 28:5-35. 
17. Spaide RF, Klancnik JM Jr. Fundus autofluorescence and central serous chorioretinopathy. Ophthalmology 2005; 112:825-833.

18. lida $\mathrm{T}$, Hagimura $\mathrm{N}$, Sato $\mathrm{T}$, et al. Evaluation of central serous chorioretinopathy with optical coherence tomography. Am J Ophthalmol 2000; 129:16-20.

19. Matsumoto H, Kishi S, Otani T, Sato T. Elongation of photoreceptor outer segment in central serous chorioretinopathy. Am J Ophthalmol 2008; 145:162-168.

20. Ojima $Y$, Hangai $M$, Sasahara $M$, et al. Three-dimensional imaging of the foveal photoreceptor layer in central serous chorioretinopathy using high-speed optical coherence tomography. Ophthalmology 2007; 114:2197-2207.

21. lijima H, Imai M, Gohdo T, Tsukahara S. Optical coherence tomography of idiopathic polypoidal choroidal vasculopathy. Am J Ophthalmol 1999; 127:301-305.

22. lijima H, lida T, Imai M, Tsukahara S. Optical coherence tomography of orange-red subretinal lesions in eyes with idiopathic polypoidal choroidal vasculopathy. Am J Ophthalmol 2000; 129:21-26.

23. Sato T, Kishi S, Watanabe G, Matsumoto H, Mukai R. Tomographic features of branching vascular networks in polypoidal choroidal vasculopathy. Retina 2007; 27:589-594.

24. Kameda T, Tsujikawa A, Otani A, et al. Polypoidal choroidal vasculopathy examined with en face optical coherence tomography. Clin Experiment Ophthalmol 2007; 35:596-601. 
25. Kon Y, lida T, Maruko I, Saito M. The optical coherence tomography-ophthalmoscope for examination of central serous chorioretinopathy with precipitates. Retina 2008; 28:864-869.

26. Tsujikawa A, Sasahara M, Otani A, et al. Pigment epithelial detachment in polypoidal choroidal vasculopathy. Am J Ophthalmol 2007; 143:102-111.

27. Ojima $Y$, Hangai $M$, Sakamoto $A$, et al. Improved visualization of polypoidal choroidal vasculopathy lesions using spectral-domain optical coherence tomography. Retina 2009; 29:52-59.

28. Matsuoka M, Ogata N, Otsuji T, et al. Expression of pigment epithelium derived factor and vascular endothelial growth factor in choroidal neovascular membranes and polypoidal choroidal vasculopathy. $\mathrm{Br} \mathrm{J}$ Ophthalmol 2004;88:809-815.

29. Marmor MF. Mechanisms of fluid accumulation in retinal edema. Doc Ophthalmol 1999; 97:239-249. 
Figure Legends

Figure 1. A 64-year-old man with polypoidal choroidal vasculopathy (PCV) and a 7-day history of rapidly decreased visual acuity in the right eye. Visual acuity was 0.09. A. Funduscopic examination shows fibrin, subretinal fluid and reddish-orange nodules with a yellow-white exudate. B. and C. Simultaneously obtained fluorescein angiography (FA) (B) and indocyanine green angiography (IA) (C) images. IA demonstrates a small branching vascular network terminating in polypoidal lesions. D. The speckle-noise-reduced spectral domain optical coherence tomography (SD-OCT) image (horizontal section corresponding to the arrow indicated in C) shows a hyperreflective shadow, suggesting fibrin (arrow), around the nodules. E. Magnified view of D. Intense reflectivity due to fibrin infiltration (arrow) is seen in the outer retina, especially between the photoreceptor outer segments (OS) and the external limiting membrane (ELM), overlying the fibrin exudate in the fovea (asterisk). $\mathrm{ONL}=$ outer nuclear layer

Figure 2. A 73-year-old man with polypoidal choroidal vasculopathy (PCV) and a several-month history of decreased visual acuity in the right eye. Visual acuity was 0.5. A. Funduscopic examination shows fibrin, subretinal fluid, and reddish-orange nodules with a yellow-white exudate. B. and C. Simultaneously obtained fluorescein angiography (FA) (B) and indocyanine green angiography (IA) (C) images. IA demonstrates a small branching vascular network terminating in polypoidal lesions. D. The speckle-noise-reduced spectral domain 
optical coherence tomography (SD-OCT) image (vertical section corresponding to the arrow indicated in C) shows a hyperreflective shadow, suggesting fibrin (arrow) in the subretinal space. E. Magnified view of D. Although fibrin is seen beneath the fovea, minimal intense reflectivity due to fibrin infiltration is present in the photoreceptor inner segments (IS) in the fovea. ONL = outer nuclear layer, $E L M=$ external limiting membrane, $O S=$ photoreceptor outer segment

Figure 3. A 78-year-old man with polypoidal choroidal vasculopathy (PCV) and a 5 day-history of rapidly decreased visual acuity in the right eye. Visual acuity was 0.1. A. Funduscopic examination shows subretinal hemorrhage and hemorrhagic pigment epithelial detachments (PEDs). B. and C. Simultaneously obtained fluorescein angiography (FA) (B) and indocyanine green angiography (IA) (C) images. D. The speckle-noise-reduced spectral domain optical coherence tomography (SD-OCT) image (vertical section corresponding to the arrow indicated in C) shows a hyperreflective shadow, suggesting subretinal hemorrhage (arrow) and hemorrhagic PED (arrowhead). E. Magnified view of D. Intense reflectivity due to hemorrhagic infiltration (arrow) is seen in the outer retina, especially between the photoreceptor outer segments (OS) and the external limiting membrane (ELM), above the subretinal hemorrhage and involves the fovea (asterisk). ONL = outer nuclear layer 


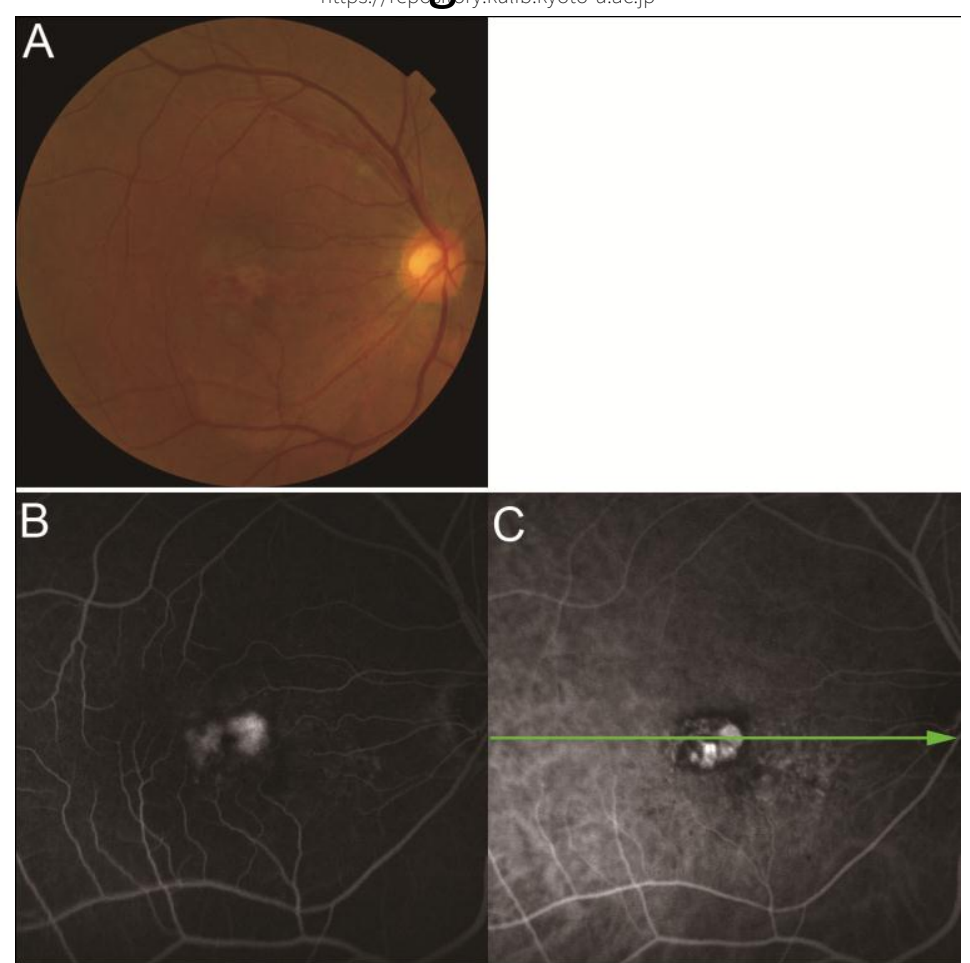

D

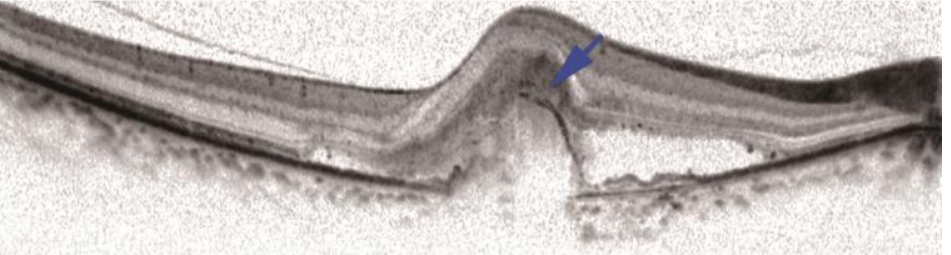

E ONL

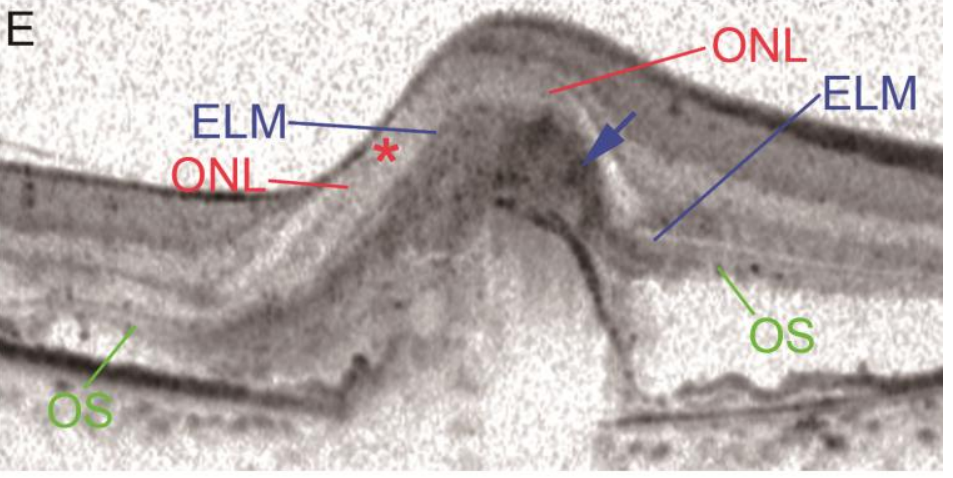



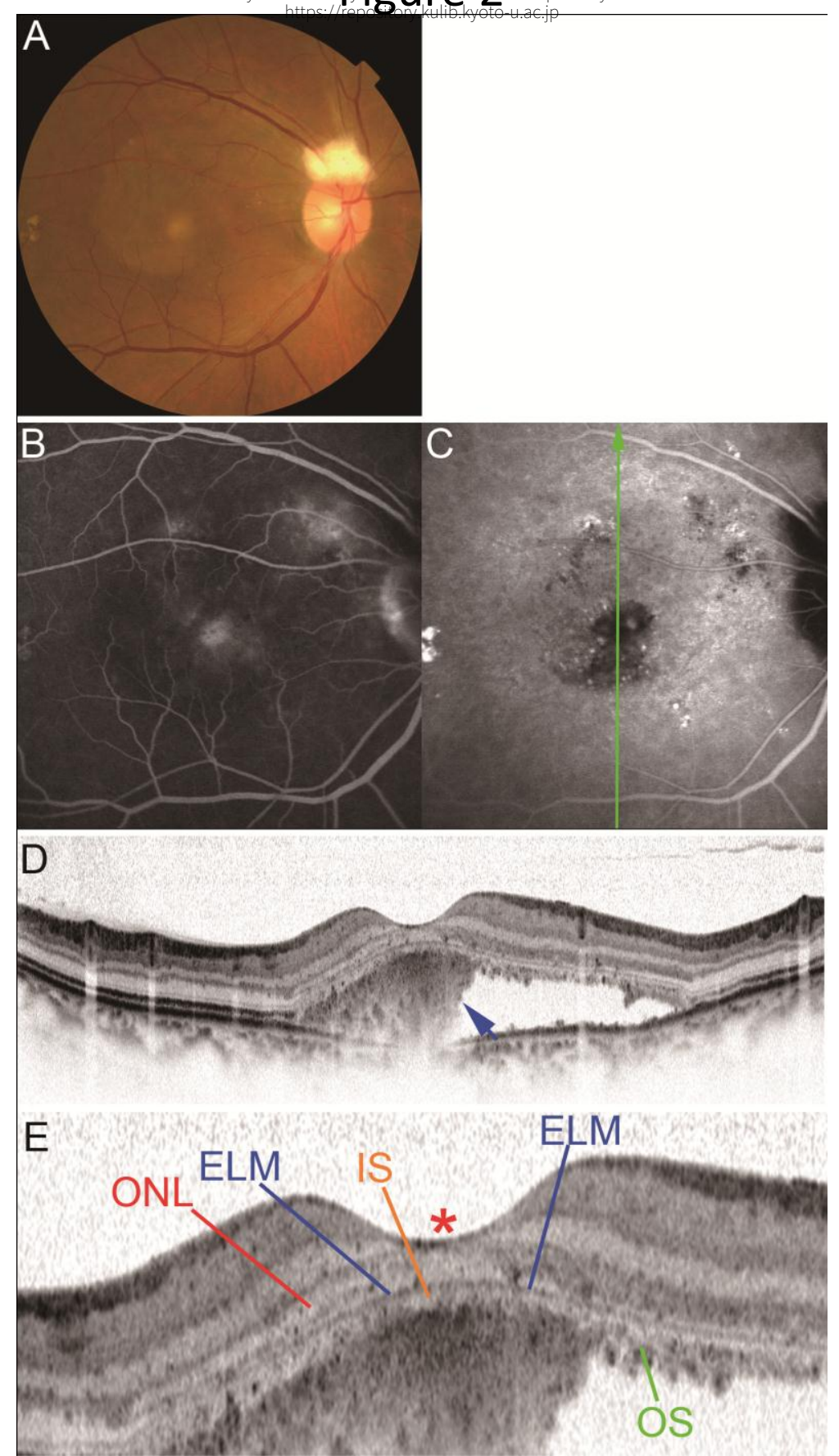


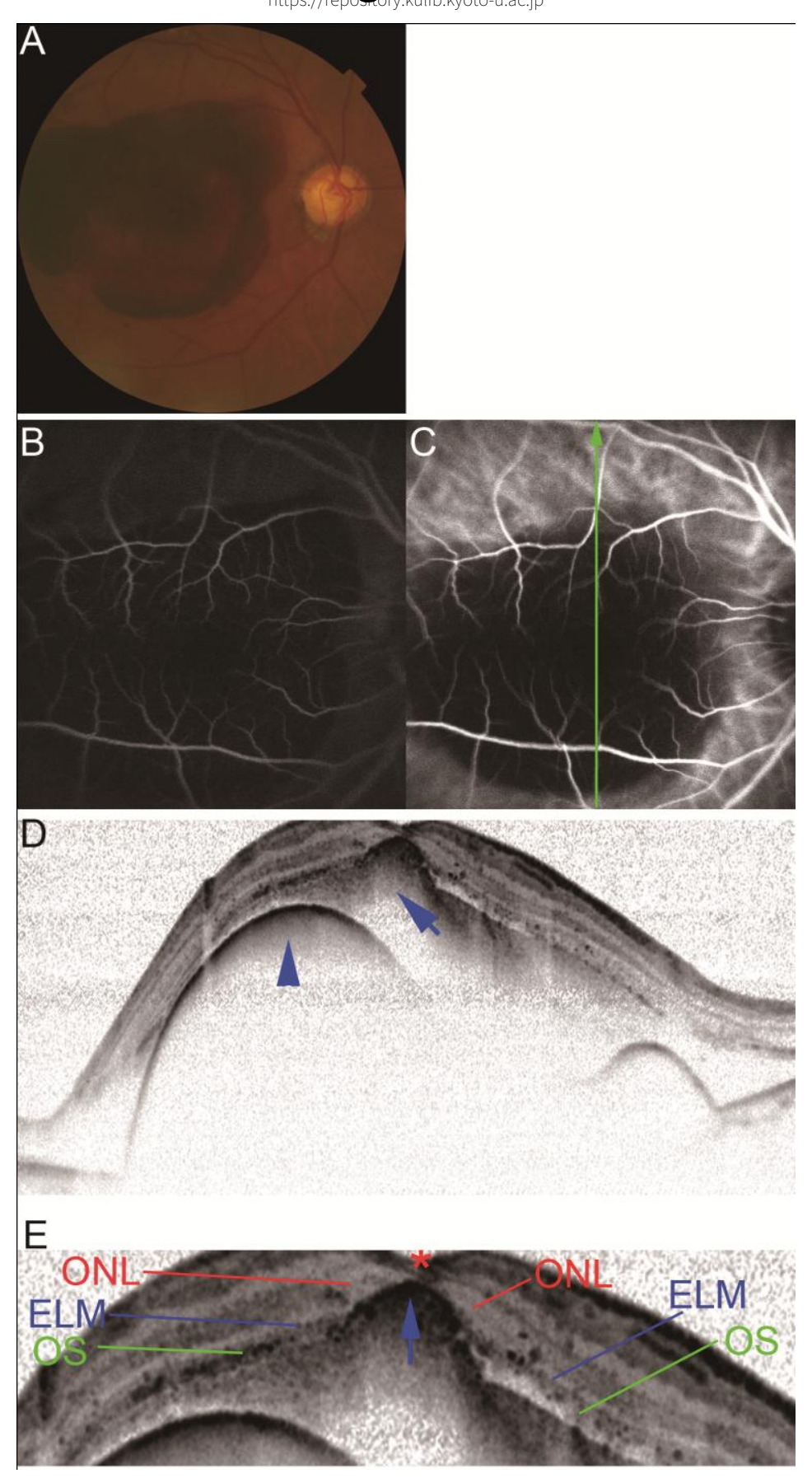

